\title{
Corrigendum: Associations of Delay in Doctor Consultation With COVID-19 Related Fear, Attention to Information, and Fact-Checking
}

\section{OPEN ACCESS}

Edited by:

Simon Ching Lam,

Tung Wah College, Hong Kong,

SAR China

Reviewed by:

Marques Shek Nam Ng

The Chinese University of Hong

Kong, China

Hilda Ho,

Children's Hospital of Orange County United States

*Correspondence: Sai-Yin Ho

syho@hku.hk

tThese authors share first authorship

Specialty section:

This article was submitted to Public Mental Health,

a section of the journal

Frontiers in Public Health

Received: 03 January 2022

Accepted: 18 January 2022

Published: 10 February 2022

Citation:

Lai AY-K, Sit SM-M, Wu SY-D, Wang $M-P$, Wong BY-M, Ho S-Y and

Lam T-H (2022) Corrigendum:

Associations of Delay in Doctor

Consultation With COVID-19 Related

Fear, Attention to Information, and

Fact-Checking.

Front. Public Health 10:847603. doi: 10.3389/fpubh.2022.847603
Agnes Yuen-Kwan Lai ${ }^{1 \dagger}$, Shirley Man-Man Sit ${ }^{1,2 \dagger}$, Socrates Yong-Da Wu ${ }^{1}$, Man-Ping Wang ${ }^{1}$, Bonny Yee-Man Wong ${ }^{2}$, Sai-Yin Ho ${ }^{2 *}$ and Tai-Hing Lam ${ }^{2}$

${ }^{1}$ School of Nursing, The University of Hong Kong, Hong Kong, Hong Kong SAR, China, ${ }^{2}$ School of Public Health, The University of Hong Kong, Hong Kong, Hong Kong SAR, China

Keywords: COVID-19, coronavirus, infodemic, infodemiology, delay in doctor consultation, patient delay, public health, information and communication technologies

\section{A Corrigendum on}

Associations of Delay in Doctor Consultation With COVID-19 Related Fear, Attention to Information, and Fact-Checking

by Lai, A. Y-K., Sit, S. M-M., Wu, S. Y-D., Wang, M-P., Wong, B. Y-M., Ho, S-Y., and Lam, T-H. (2021). Front. Public Health 9:797814. doi: 10.3389/fpubh.2021.797814

In the original article, there was an error. The incorrect adjusted odds ratio was included in the abstract for the association between fact-checking and delay.

A correction has been made to Abstract, Results, 1:

The incorrect text stated: Results: Of 4,551 respondents (46.5\% male, 59.7\% aged over 45 years), $10.1 \%$ reported delay in doctor consultation. The mean score was 6.4 for fear, 8.0 for attention and 7.4 for fact-checking. Delay was more common in males and increased with age and fear. High vs. low level of fear was associated with delay [adjusted odd ratios (AOR) 2.68, 95\% confidence interval (CI) 2.08, 3.47]. Moderate level of fact-checking was negatively associated with delay (AOR $1.28,95 \%$ CI $0.98,1.67)$. Females reported greater fear and fear decreased with age. Fear increased with attention to information and decreased with fact-checking. Fear substantially mediated the association of delay with attention (96\%) and fact-checking (30\%).

The corrected text appears below:

Of 4,551 respondents ( $46.5 \%$ male, $59.7 \%$ aged over 45 years), $10.1 \%$ reported delay in doctor consultation. The mean score was 6.4 for fear, 8.0 for attention and 7.4 for fact-checking. Delay was more common in males and increased with age and fear. High vs. low level of fear was associated with delay [adjusted odd ratios (AOR) 2.68, 95\% confidence interval (CI) 2.08, 3.47]. Moderate level of fact-checking was negatively associated with delay (AOR 0.72, 95\% CI 0.56, 0.92). Females reported greater fear and fear decreased with age. Fear increased with attention to information and decreased with fact-checking. Fear substantially mediated the association of delay with attention (96\%) and fact-checking (30\%).

The authors apologize for this error and state that this does not change the scientific conclusions of the article in any way. The original article has been updated. 
Publisher's Note: All claims expressed in this article are solely those of the authors and do not necessarily represent those of their affiliated organizations, or those of the publisher, the editors and the reviewers. Any product that may be evaluated in this article, or claim that may be made by its manufacturer, is not guaranteed or endorsed by the publisher.
Copyright (C) 2022 Lai, Sit, Wu, Wang, Wong, Ho and Lam. This is an open-access article distributed under the terms of the Creative Commons Attribution License (CC $B Y)$. The use, distribution or reproduction in other forums is permitted, provided the original author(s) and the copyright owner(s) are credited and that the original publication in this journal is cited, in accordance with accepted academic practice. No use, distribution or reproduction is permitted which does not comply with these terms. 